\title{
An Effective Approach For Car Driving In Mountain Area Using Optimal Regulator
}

\author{
Nirmalya Chandra ${ }^{1}$, Dhritimoy Roy ${ }^{2}$, Avinash Kumar ${ }^{3}$, Abhik Ghosh ${ }^{4}$ \\ Assistant Professor, Birbhum Institute of Engineering and Technology, Suri, Birbhum ${ }^{1}$ \\ Students, Birbhum Institute of Engineering and Technology, Suri, Birbhum ${ }^{2,3,4}$
}

\begin{abstract}
Optimal Control is applied on the basis of an effective approach for car driving in mountain area. The report will review the original project of an effective approach for car driving in mountain area. Then, Optimal Control will be investigated by applying several design concepts with detailed comments about each design applied. The thesis derives a formulation of the out-of-sequence measurements (OOSM) problem for nonlinear state-space models when developing a Car driving in the mountain area automatically. In this paper we have to try an approach how the total constraints effect on the path of car driving should be minimize.
\end{abstract}

Keywords: Optimal Regulator, OOSM logic, Hamilton-Jacobi-Bellman equations, Riccati equation, Minimum Time Solution, LQR problem

\section{INTRODUCTION}

Optimal control theory is a mature mathematical discipline proud. Current research suggests the use of linear with numerous applications in both science and quadratic performance index for optimal control engineering. Optimal control deals with the problem of regulators in various application. Some examples include finding a control law for a given system such that a certain correcting the trajectory of rocket and air vehicles, optimality criterion is achieved. A control problem vibration suppression of flexible structures, and airplane includes a cost functional that is a function of state and stability. The focus of all these cases is in suppressing control variables. An optimal control is a set of differential system deviations rapidly[5]. In the current work we have equations describing the paths of the control variables that try to approach a design of optimal regulator into car minimize the cost function. The minimization of cost driving system in the mountain area.

function on the basis of Linear Quadratic Regulator problem in optimal control can be derived using by solving the Hamilton-Jacobi-Bellman equation. For control system gain scheduling consider as a mapping from process parameter to control parameter in which model reference adaptive control is used into a closed loop control for up-gradation of the response parameters to create the desire model. MIT rule is use for adjustment of the parameters up-gradation .MIT rule given below,

$\frac{d \theta}{d t}=-\gamma \frac{\delta J}{\delta \theta}=-\gamma e \frac{\delta e}{\delta \theta}$

$J(\theta)=1 / 2 e^{2}(\theta)$

\section{PROJECT SUMMERY}

Optimal Control is applied on the basis of an effective approach for car driving in mountain area. The report will review the original project of an effective approach for car driving in mountain area. Then, Optimal Control will be investigated by applying several design concepts with detailed comments about each design applied. A conclusion of the work will end the report giving future recommendations and comments. This thesis covers areas within estimation and optimal control of vehicles, in particular four-wheeled vehicles.

After more than three hundred years of evolution, optimal controltheory has been developed to the based on theoretical foundation laid by generation of mathematician. The maximum principal developed in the late 1950 by Pontryagin and his coworkers [1] , is among the biggest successes in optimal control. The linear analysis approach is considered on a transformation general non linear form for a dynamic system into a linear system for the analysis of controller design [2] .Now, linear quadratic optimal problem based on dynamic compensation is considered for a general quadratic performance index [3]. Recently modified Riccati equation has already been associated with tracking a target under measurement uncertainty [4]. In this paper some properties of the modified Riccati equation will be derived and

One topic is how to handle delayed and out-of-sequence measurements (OOSMs) in tracking systems. The algorithms improve estimation accuracy and tracking robustness, compared with methods that do not utilize the linear substructure. Optimization-based regulator control methods have found their way into automotive applications. This control for vehicles typically results in control inputs that give aggressive maneuvering. Another conclusion is that the optimal driving techniques are different depending on tire-road characteristics. The conclusions motivate the design of a novel, two-level hierarchical approach to optimal trajectory generation for wheeled vehicles. Here we consider a basic block diagram for a Non linear control system where the State Estimators is connected as a control parameter between both the plant input and output. In the given diagram shown below :- $x(t)$ 
denotes the state of the system in time ' $\mathrm{t}$ ' ; $y(t)$ represent the system response at ' $t$ '; $u(t)$ is the system input ;

$$
u(t)=K^{\prime}(t) x(t)
$$

Now the change of the state equation is described as

$$
x(t)=F(t) x(t)+G(t) u(t)
$$

$$
y(t)=H^{\prime}(t) x(t)
$$

where $F(t), G(t), H(t)$ are the matrix functions of time with continuous entries.

The Transfer Function of the given state equations using Matrixes is denoted by $W(s)$ which is calculated below :-

$W(s)=H^{\prime}(s I-F)^{-1} G$

In our project we try to implement an effective approach for minimization the upcoming constrains badly effects on the path of a driving car in mountain area as per our best effort. The details method will discussed later in the corresponding chapter of the project report.

\section{BLOCK DIAGRAM}

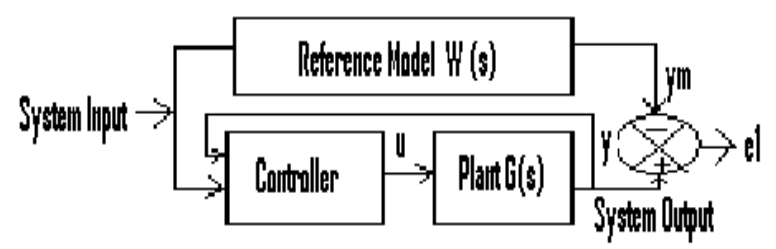

Fig1: Model Reference Adaptive Feedback Arrangement

\section{HAMILTON-JACOBI-BELLMAN EQUATIONS}

We now turn to optimal control problems where the state $x$ $\in R^{n_{x}}$ and control $u \in U(x) \subseteq R^{n_{u}}$ are real-valued vectors. To simplify notation we will use the shortcut $\min _{\mathrm{u}}$ instead of $\min _{u \in U(x)}$, although the latter is implied unless noted otherwise. Consider the stochastic differential equation

$d x=f(x, u) d t+F(x, u) d w$

where $d$ wis $n_{w}$-dimensional Brownian motion. This is sometimes called a controlled Ito diffusion, with $f$ $(x, u)$ being the drift and $\mathrm{F}(x, u)$ the diffusion coefficient. In the absenceof noise, i.e. when $\mathrm{F}(x, u)=0$, we can simply write $\dot{x}=f(x, u)$. However in the stochasticcase this would be meaningless because the sample paths of Brownian motion are notdifferentiable (the term $d w / d t$ is infinite). What equation (2.2) really means is that theintegral of the left hand side is equal to the integral of the right hand side: $x(t)=x(0)+\quad \int_{0}^{t} f(x(s), u(s)) d s \int_{0}^{t} F(x(s), u(s)) d w$

The last term is an Ito integral, de.ned for squareintegrable functions $g(t)$ as $\int_{0}^{t} G(s) d w(s)=\lim _{n \rightarrow \infty} \sum_{k=0}^{n-1} G\left(s_{k}\right)\left(w\left(s_{k+1}\right)-w\left(s_{k}\right)\right)$ (8)

where $0=s_{0}<s_{2}<\ldots \ldots<s_{n}=t$

We will stay away from the complexities of stochastic calculus to the extent possible. Instead we will discretize the time axis and obtain results for the continuous-time case in the limit of infinitely small time step. The appropriate Euler discretization of (6) is

$x_{k+1}=x_{k}+\Delta f\left(x_{k}, u_{k}\right)+\sqrt{\Delta} F\left(x_{k}, u_{k}\right) \epsilon_{k}$

where $\Delta$ is the time step, $\epsilon_{k} \sim N\left(0, I^{n_{w}}\right)$ and $x_{k}=x(k \Delta)$.

The' $\sqrt{\Delta}$ 'term appears becausethe variance of Brownian motion grows linearly with time, and thus the standard deviationof the discrete-time noise should scale as $\sqrt{\Delta}$. To define an optimal control problem we also need a cost function. In finite-horizon problems, i.e. when a final timet $_{\mathrm{f}}$ is specified, it is natural to separate the total costinto a time-integral of a cost rate $\mathrm{l}(\mathrm{x}, \mathrm{u}, \mathrm{t}) \geq 0$, and a final cost $h$ $(x) \geq 0$ which is onlyevaluated at the final state $x\left(t_{f}\right)$. Thus the total cost for a given state-control trajectory

$\left\{x(t), u(t): 0 \leq t \leq t_{f}\right\}$ is defined as

$J(x(\cdot), u(\cdot))=h\left(x\left(t_{f}\right)\right)+\int_{0}^{t_{f}} \iota(x(t), u(t), t) d t$ (10)

Keep in mind that we are dealing with a stochastic system. Our objective is to find a controllaw $u=\pi(x, t)$ which minimizes the expected total cost for starting at a given $(x$, $t$ )andacting according $) \pi$ thereafter. In discrete time the total cost becomes

$J(x ., u)=.h\left(x_{n}\right)+\Delta \sum_{k=0}^{n-1} \iota\left(x_{k}, u_{k}, k \Delta\right)$

Where $=t_{f} / \Delta$ is the number of time steps (assume that $t_{f} / \Delta$ is integer).

\section{RICCATI EQUATION}

Relation with linear equations :

Consider the Riccati equation(with time-varying coefficient matrices)

$-\dot{P}=P F+F^{\prime} P-P G R^{-1} G^{\prime} P+Q P(T, T)=A$

and the linear equation

$$
\left[\begin{array}{l}
\dot{X} \\
\dot{Y}
\end{array}\right]=\left[\begin{array}{cc}
F & -G R^{-1} G^{\prime} \\
-Q & -F^{\prime}
\end{array}\right]\left[\begin{array}{l}
X \\
Y
\end{array}\right] \quad\left[\begin{array}{l}
X(T) \\
Y(T)
\end{array}\right]=\left[\begin{array}{l}
I \\
A
\end{array}\right]
$$

Then the solution of $(11)$ exists on $\left[t_{0}, T\right]$ if $X(t)$ is nonsingular on $\left[t_{0}, T\right]$.

$P(t, T)=Y(t) X^{-1}(t)$

Conversely, if the solution of $(\sigma)$ exists on $[t o, T]$, and $\theta(\mathrm{t}, \mathrm{s})$ denotes the transition matrix of $\dot{x}(t)=[F(t)-G$ $(t) R^{-1}(t) G$ ' $\left.(t) P(t)\right] x(t)$, then $X(t)=\theta(t, T), Y(t)=P(t) \theta(t$, $T)$ is the solution of (7), with $X(t)$ nonsingular on [to, T], and with (8) holding. This again is straight forward to 
verify. If $\varphi(t, s)$ is the $2 n \times 2 n$ transition matrix associated with (7) and it is partitioned into four $\mathrm{n} \times n$ sub matrices, then

$P(t, T)=\left[\varphi_{21}(t, T)+\varphi_{22}(f, T) A\right]\left[\varphi_{11}(t, T)+\varphi_{12}(t, T) A\right]^{-1}$ ........ (15)

\section{DYNAMIC PROGRAMMING}

Optimization problems such as the one stated above are efficiently solved via dynamic programming (DP). DP relies on the following obvious fact: if a given state-action sequence is optimal, and we were to remove the first state and action, the remaining sequence is also optimal (with the second state of the original sequence now acting as initial state). This is the Bellman optimality principle. Note the close resemblance to the Markov property of stochastic processes (a process is Markov if its future is conditionally independent of the past given the present state). The optimality principle can be reworded in similar language: the choice of optimal actions in the future is independent of the past actions which led to the present state. Thus optimal state-action sequences can be constructed by starting at the final state and extending backwards. Key to this procedure is the optimal value function(or optimal cost-to-go function) $v(x)=$ "minimal total cost for completing the task starting from state $x$ ". This function captures the long-term cost for starting from a given state, and makes it possible to find optimal actions through the following algorithm:

Consider every action available at the current state, add its immediate cost to the optimal value of the resulting next state, and choose an action for which the sum is minimal. The above algorithm is "greedy" in the sense that actions are chosen based on local information, without explicit consideration of all future scenarios. And yet the resulting actions are optimal. This is possible because the optimal value function contains all information about future scenarios that is relevant to the present choice of action. Thus the optimal value function is an extremely useful quantity, and indeed its calculation is at the heart of many methods for optimal control. The above algorithm yields an optimal action $\mathrm{u}=\pi(\mathrm{x}) \in \mathrm{U}(\mathrm{x})$ for every state $\mathrm{x}$. A mapping from states to actions is called control law or control policy. Once we have a control law $\pi: X \rightarrow U(X)$ we can start at any state $\mathrm{x}_{0}$, generate action $\mathrm{u}_{0}=\pi\left(\mathrm{x}_{0}\right)$, transition to state $x_{1}=$ next $\left(x_{0}, u_{0}\right)$, generate action $u_{1}=$ $\pi\left(x_{1}\right)$, and keep going until we reach $x^{\text {dest }}$ Formally, an optimal control law $\pi$ satisfies

$\pi(x)=\quad \arg \quad \min _{u \in U(x)}\{\quad \operatorname{cost}(x, u)+v(n \operatorname{ext}(x ; u))$
$\ldots . .(14)$

The minimum in (8) may be achieved for multiple actions in the set $U(x)$, which is why $\pi$ may not be unique. However the optimal value function vis always uniquely de.ned, and satisfies
$v(x)$
$\operatorname{cost}(x, u)+v(n e x t$
$(x ;$

Equations (3.1) and (3.2) are the Bellman equations. If for some $\mathrm{x}$ we already know $v($ next $(x, u))$ for allu $\in U(x)$, then we can apply theBellman equations directly and compute $u(x)$ and $v(x)$. Thus dynamic programming is particularly simple in acyclic graphs where we can start from $x^{\text {dest }}$ with $v\left(x^{\text {dest }}\right)=0$, and perform a backward pass in which every state is visited after all its successor states have been visited. It is straightforward to extend the algorithm to the case where we are given non-zero final costs for a number of destination states (or absorbing states).

\section{PONTRYAGIN'S PRINCIPLE}

Optimal control theory is based on two fundamental ideas. One is dynamic programming and the associated optimality principle, introduced by Bellman in the United States. The other is the maximum principle, introduced by Pontryagin in the Soviet Union. The maximum principle applies only to deterministic problems, and yields the same solutions as dynamic programming. Unlike dynamic programming, however, the maximum principle avoids the curse of dimensionality.

\section{MINIMUM TIME SOLUTION}

Since the main purpose of the controller is to suppress vibrations in minimum time, the time for the system to come to rest is taken as the objective function. A functional expression for this can be written

$$
I=\int_{0}^{T} d t, T=\min
$$

subject to $d x / d t=A x+b f, x(0)=x o, x(T)=0$ with control force limits

$$
\left|f_{i}\right| \leq F_{i}, \quad i=1,2, \ldots, p \text {. }
$$

This problem can be written in short form as

$\operatorname{Imin}=\int_{0}^{T} d t, d x / d t=A x+b f, x(0)=x o, x(T)=0,|f| \leq F$, (19)

where $x$ is the state vector of dimension $2 n . A$ is the $2 n \times 2 n$ plant matrix, $B$ is $2 \mathrm{n} \times p$ control matrix, $f$ isthe control force vector of dimension $p, x(0)$ is the initial state vector, and $x(T)=0$ is the final state ofthe system. Bo, for this problem can be written as

$$
B_{0}=1 \cdot \Sigma\left(\hat{\partial} \psi / \hat{\alpha}_{i}\right)\left(d x_{i} / d t\right)-(\hat{\partial} \psi / \partial t)
$$

$$
\text { Where } \mathrm{i}=1,2, \ldots \ldots, \mathrm{n} \text {; }
$$

\section{ORIGINAL PROJECT OVERVIEW}

In this section of the report, first, brief discussion of original project . In this section of the report, first, brief discussion of original project. This thesis addresses topics within nonlinear estimation and optimal control of ground vehicles. The ever-continuing advancements in computing power, sensors, and control theory, have led to an increased interest in autonomous vehicles, illustrated by, for example, the Google car. The inclusion of more sensors gives potential for better estimation and 
understanding of the vehicle motion, which makes it possible to formulate control principles for improved autonomy. On the other hand, more sensor measurements, arriving with different delays and accuracy, increase demands on the system that is responsible for combining the sensor signals.

\section{OPTIMAL CONTROL OF GROUND VEHICLES:}

Optimal control of ground vehicles is interesting for several reasons. One objective is to develop improved active[7] safety and driver-assistance systems for production cars.

\section{A. GROUND-TIRE INTERACTION:}

The dominant interaction between a ground vehicle and its surroundings is via the tires; it is the friction forces between the tires and ground that generate most of the vehicle motion. See below the figure, where the tilt about the wheel $\mathrm{x}$-axis has been ignored. The wheel has longitudinal velocity $\mathrm{v}^{\mathrm{x}}$ with respect to an inertial system, resolved in the wheel's coordinate system, and the normal load $\mathrm{F}^{\mathrm{z}}$ acts on the wheel. A torque balance around the center of the wheel gives,

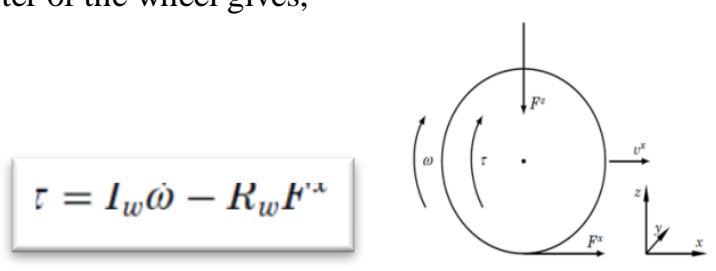

A wheel model for longitudinal dynamics. The input torque $\tau$ generates a longitudinal friction force , $\mathrm{F}^{\mathrm{x}}$ which gives rise to angular velocity $\mathrm{w}$ and longitudinal wheel velocity $v^{x}$

\section{ALGORITHM OF DRIVE AN} AUTOMATIC CAR DOWN IN A MOUNTAIN:

Step 1: Shift into second gear before starting down a mountain. Reduce speed before downshifting so you don't damage the transmission.

Step 2: Obey the posted speed limit or "Maximum Safe Speed" limit. Look for steep hill warning signs.

Step 3: Maintain a safe speed for road conditions. Resist the temptation to "zoom" down the mountain.

Step 4: Apply the brakes once you reach a safe speed. For example, if the safe speed is 40 , gently apply the brakes when you are traveling 40 miles per hour to reduce your speed to 35 miles per hour. Release the brakes. Repeat this until you reach the bottom of the mountain. If you need to reduce speed further on a steep downgrade, apply the brakes and downshift into first gear.

Step 5: Shift into drive once you are back on flat land. Use your brakes for routine slowing and stopping.

\section{INCLINED PULL REQUIRED TO MOVE A BODY}

Down An Inclined Plane :

When the angle of inclination $(\beta)$ of the inclined plane is less than the angle of repose, external force is necessary to move a body down an inclined plane.

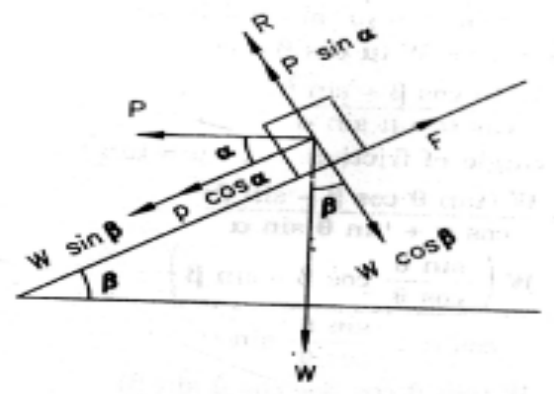

Let,

$P=$ required pull applied at an angle $\alpha$ with the inclined plane,

$F=$ limiting friction,

$W=$ weight of the body,

$R=$ normal reaction of the inclined plane on the body,

$B=$ angle of inclination of the inclined plane with the Horizontal.

The body is in equilibrium under the action of the following forces:

1) ' $W$ ' acting vertically downward through C.G. of the body,

2) ' $P$ ' applied externally to move the body down the inclined plane,

3) ' $R$ ' acting along the inclined plane, and

4) ' $F$ ' acting along the inclined in a direction opposite to that in which the body has a tendency to move.

Therefore,

(a) $\quad \sum X_{A}=0$, and (b) $\sum Y_{P}=0$ where

$\sum X_{A}=$ algebraic sum of the resolved parts of the forces

along the inclined plane, and

$\sum Y_{P}=$ algebraic sum of the resolved parts of the forces along a direction perpendicular to the inclined plane.

Now $\sum X_{A}=F-W \sin \beta-P \cos \alpha$

and $\sum Y_{P}=R+P \sin \alpha-W \cos \beta$

According to condition (a), we get

$$
F-W \sin \beta-P \cos \alpha=0
$$

or, $\mu R-W \sin \beta-P \cos \alpha=0$

or, $P \cos \alpha=\mu R-W \sin \beta$

According to condition (b), we get

$R+P \sin \alpha-W \cos \beta=0$

or, $R=W \cos \beta-P \sin \alpha$

Putting this value of $\mathrm{R}$ in equation (4.3) we get,

$P \cos \alpha=\mu(W \cos \beta-P \sin \alpha)-W \sin \beta$

$$
=\mu W \cos \beta-\mu P \sin \alpha-W \sin \beta
$$

or, $\quad P(\cos \alpha+\mu \sin \alpha)=W(\mu \cos \beta-\sin \beta)$

$P=\quad W(\mu \cos \beta-\sin \beta)$

$(\cos \alpha+\mu \sin \alpha)$

Let, $\theta=$ angle of friction. Therefore $\mu=\tan \theta$ 


$P=\frac{\frac{W(\tan \theta \cos \beta-\sin \beta)}{(\cos \alpha+\tan \theta \sin \alpha)}}{\frac{W(\sin \theta \cos \beta-\cos \theta \sin \beta)}{\cos \theta}}$
$\frac{\frac{(\cos \alpha \cos \theta+\sin \theta \sin \alpha)}{\cos \theta}}{P=\frac{W \sin (\theta-\beta)}{(27)}}$
$\ldots \ldots \ldots \ldots . . . \cos (\alpha-\theta)$

Drive An Automatic Car Up In A Mountain:

When approaching a steep hill, you can initially leave your vehicle in $\mathrm{D}$ and start up the hill. In most cases your transmission will downshift itself once it gets to the end of a gear, but if you feel comfortable, you can downshift the transmission yourself[8]. Usually shifting down to 2 will give you enough extra power to make it to the top of the hill with less effort. Remember never to shift into 1 if you are traveling over $30 \mathrm{mph}$. This is especially helpful if you are towing anything such as a boat or trailer. Once you reach the top of the hill, you can return the transmission to D.

\section{INCLINED PULL REQUIRED TO MOVE A BODY UP AN INCLINED PLANE}

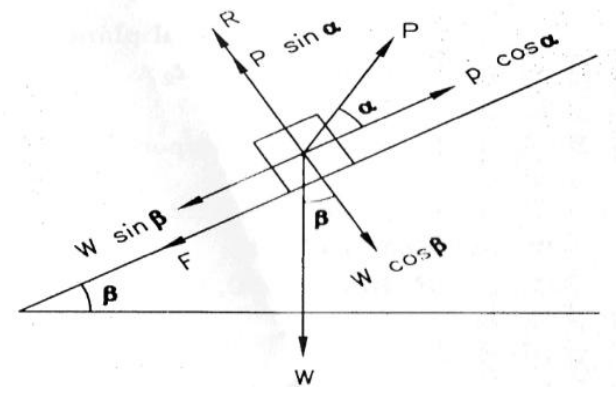

Resolved part of $\mathrm{P}$ along the inclined plane $=P \cos \alpha$

Resolved part of $\mathrm{P}$ perpendicular to the inclined plane $=P$ $\sin \alpha$

Resolved part of $\mathrm{W}$ perpendicular to the inclined plane $=$ $W \cos \beta$

Resolved part of $\mathrm{w}$ along the inclined plane $=W \sin \beta$

Resolved part of $\mathrm{F}$ along the inclined plane $=F \cos 0^{\circ}$ $=F$

Resolved part of $\mathrm{F}$ perpendicular to the inclined plane $=W$ $\sin 0^{\circ}=0$

Resolved part of $\mathrm{R}$ perpendicular to the inclined plane $=R$ $\sin 90^{\circ}=R$

Resolved part of $\mathrm{R}$ along the inclined plane $=R \cos 0^{\circ}$ $=0$
The body is in equilibrium on the inclined plane under the action of the following forces [9]:

1) ' $W$ ' acting vertically downward,

2) 'P' applied at an angle $\alpha$ with the inclined plane,

3) ' $R$ ' acting at right angles to the inclined plane,

4) ' $F$ ' acting along the inclined plane in a direction opposite to the direction in which the body has a tendency to move .

Therefore,

(a) $\sum X_{A}=0$, and (b) $\sum Y_{P}=0$ where $\sum X_{A}=$ algebraic sum of the resolved parts of the forces along the plane, and $\sum Y_{P}=$ algebraic sum of the resolved parts of the forces Along a direction perpendicular to the inclined plane.

Now ,

$\sum X_{A}=-F-W \sin \beta+P \cos \alpha$

and $\sum Y_{P}=R+P \sin \alpha-W \cos \beta$

According to condition (a), we get

$$
-F-W \sin \beta+P \cos \alpha=0
$$

or, $-\mu R-W \sin \beta+P \cos \alpha=0$

or, $P \cos \alpha=\mu R+W \sin \beta$

According to condition (b), we get

$R+P \sin \alpha-W \cos \beta=0$

or, $R=W \cos \beta-P \sin \alpha$

Putting this value of $\mathrm{R}$ in equation (30) we get,

$P \cos \alpha=\mu(W \cos \beta-P \sin \alpha)+W \sin \beta$

$=\mu W \cos \beta-\mu P \sin \alpha+W \sin \beta$

or, $\quad P(\cos \alpha+\mu \sin \alpha)=W(\mu \cos \beta+\sin \beta)$ $W(\mu \cos \beta+\sin \beta)$

$P=\frac{(\mu \cos \beta+\sin \beta)}{(\cos \alpha+\mu \sin \alpha)}$

Let, $\theta=$ angle of friction. Therefore $\mu=\tan \theta$

$$
\begin{aligned}
P & =\frac{\frac{W(\tan \theta \cos \beta+\sin \beta)}{(\cos \alpha+\tan \theta \sin \alpha)}}{\frac{W(\sin \theta \cos \beta+\cos \theta \sin \beta)}{\cos \theta}} \\
P & =\frac{\frac{W \sin (\theta+\beta)}{(\cos \alpha \cos \theta+\sin \alpha \sin \theta) / \cos \theta}}{\cos (\alpha-\theta)}
\end{aligned}
$$

XIII. MOVE A BODY ROAD BANKING IN MOUNTAIN AREA

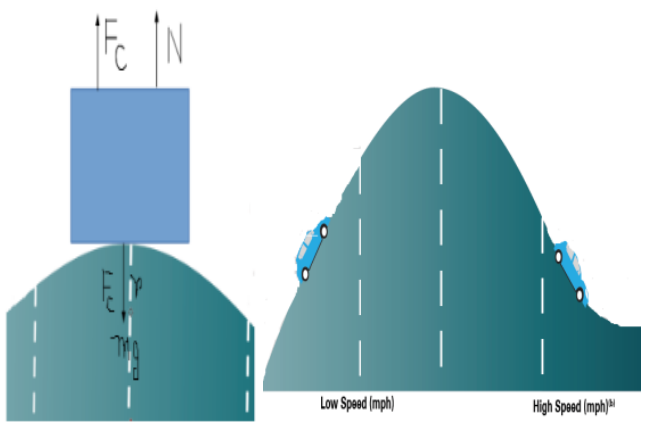


$N=m g-4 m \pi^{2} f^{2} r$

For contact in $\operatorname{road} N=0$

$m g-4 m \pi^{2} f^{2} r=0$

or, $4 m \pi^{2} f^{2} r=m g$

or, $f^{2}=g /\left(4 \pi^{2} r\right)$

$f=g /\left(4 \pi^{2} r\right)^{1 / 2}$

Now using the logical optimal regulator we try to design the path optimally and get the following outcomes where it is shown that the curve try to follows the smoothness behaviors after the processing time on output characteristic of the various side angels of the car as well as velocity control output.
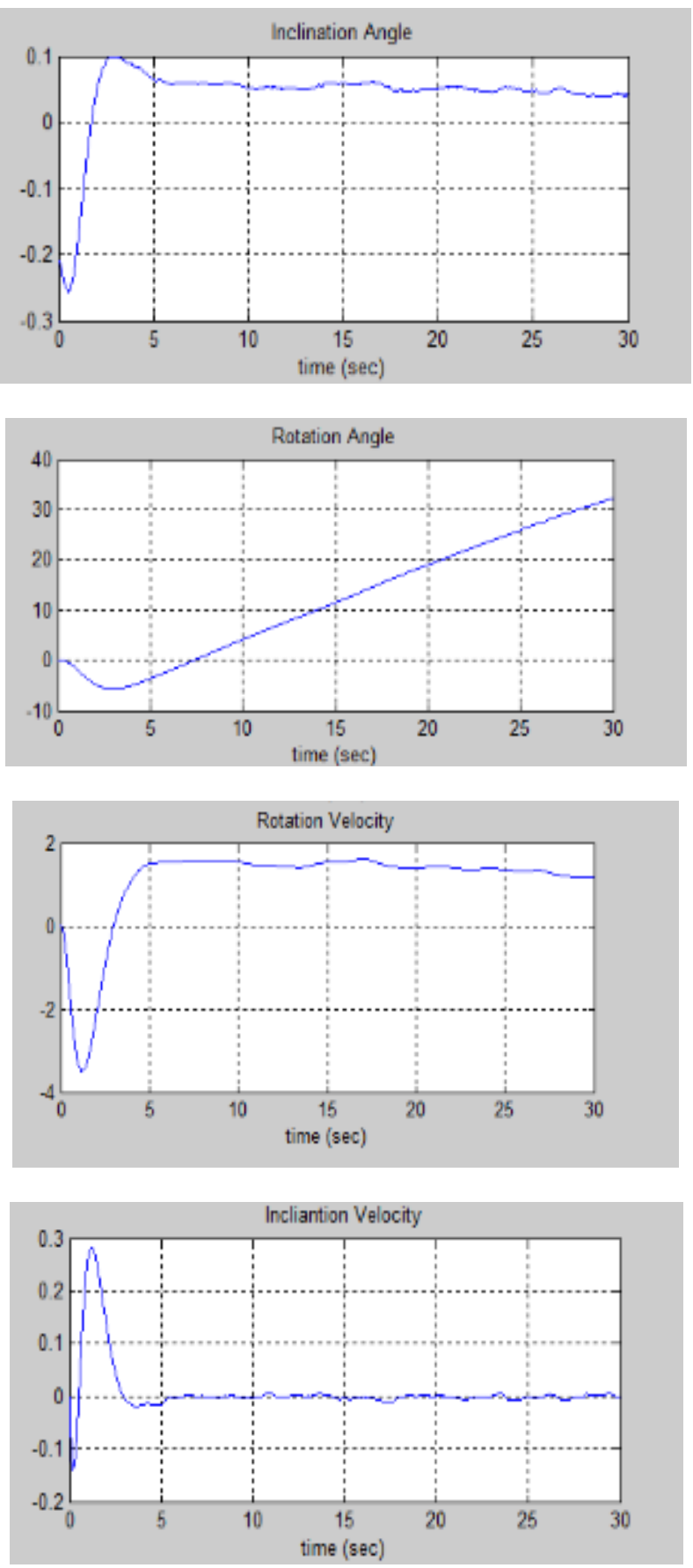

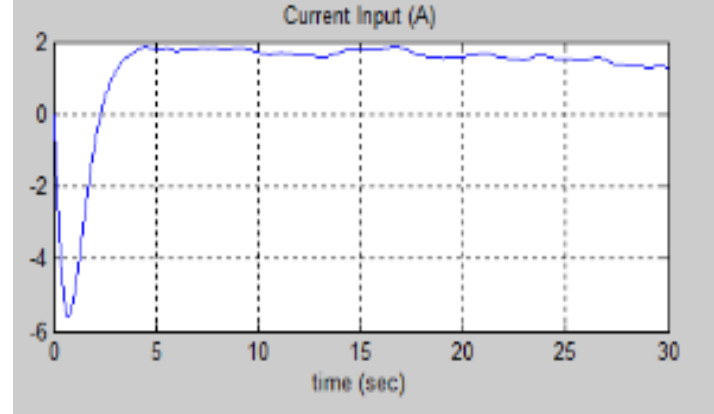

XIV. CONCLUSION

An approximation approach an optimal control is optimized by a systematic process. Hamilton - Jacobi Bellman equation shows the way which are user friendly but the corresponding Linear Quadratic Regulator(LQR) attached with it can be 3 to 6 times worse. In that case we considered here minimum time solution process.

In the $\mathrm{A}$ to $\mathrm{Z}$ process of the corresponding would be an effective approach to go ahead to the original hardware implementation . On this surcumtences are logical optimal regulator design for minimization the error as well as the interrupts fetching in the time of a car driving in mountain area is mostly demand, which is to be try to maintain through out the project.

\section{ACKNOWLEDGMENTS}

The authors would like to thank the authorities of Birbhum Institute of Engineering and Technology for providing every kind of supports and encouragement during the working process. The authors thanks to reviewers for giving us such attention and time. The authors also acknowledge the unknown referees for their valuable comments and suggestions for improvement. Last but not the least the authors are giving a vote of thanks to our nearest and dearest parents and our be loving family members for providing mentally support to us.

\section{REFERENCE}

[1] William Blake,"optimal control," Nonlinear and Dynamic Optimization: From Theory to Practice." B. Chachuat@2007 Automatic Control Laboratory, EPFL, Switzerland.

[2] J. K. Hedrick and A. Girard , "Control of Nonlinear Dynamic Systems: Theory and Applications" Copyright (C) 2010 All rights reserved

[3] G. Zhang, L. Liu and W. Liu , "Linear Quadratic Optimal Control Based On Dynamic Compensation" Received October 2010; revised March 2011.

[4] Yvo Boersand Hans Driessen ," On the modified Riccati equation and its application to target tracking ."

[5] Alexander A. Bolonkin and Robert L. Sierakowski ," Design of Optimal Regulators "San Diego, California, USA, 15-18 Sep 2003.

[6] Brian D.O. Anderson and John B. Moore ," Optimal Control Linear Quadratic Methods.” @ 1989 by Prentice-Hall, Inc. A Division of Simon \& Schuster Englewood Cliffs, NJ 07632.

[7] Karl Berntorp," Particle Filtering and Optimal Controlfor Vehicles and Robots." Fc 2014 by Karl Berntorp. All rights reserved. Printed in Sweden by Media-Tryck.Lund 2014.

[8] Admin, " Guideto driving Up and down hills ." Apr 232012

[9] A.R. Basu ," Engineering Mechanics."

[10] Mohammad Shahab and Prof. Magdi S. Mahmoud ," Optimal Control of Robotic Wheelchair." 


\section{BIOGRAPHIES}

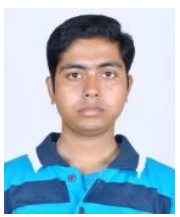

Dhritimoy Roy was born in India. He is a student in the Department of Electronics and Communication Engineering, Birbhum Institute of Engineering and Technology ( Govt. Aided Institution ), Suri, Birbhum731101 under West Bengal University of Technology, Kolkat since 2011. His currently research interest in An effective approach for car driving in mountain area using Optimal Regulator.

Avinash Kumar was born in India. She is pursuing B.Tech in Electronics and Communication Engineering from Birbhum Institute of Engineering \& Technology, Suri Affiliated to West Bengal University of Technology, Kolkata since 2011. His currently research interest in An effective approach for car driving in mountain area using Optimal Regulator.

Abhik Ghosh was born in India. He is pursuing B.Tech in Electronics and Communication Engineering from Birbhum Institute of Engineering \& Technology, Suri Affiliated to West Bengal University of Technology, Kolkata since 2011. His currently research interest in An effective approach for car driving in mountain area using Optimal Regulator.

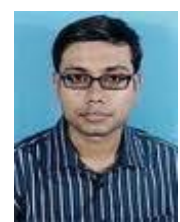

Nirmaly a Chandra was born in India. He has been an Assistant Professor in the Department of Electronics and Communication Engineering, Birbhum Institute of Engineering and Technology ( Govt. Aided Institution ), Suri, Birbhum731101. His currently research interest in Control System, Adhoc Networks, Digital Modulation, Digital Signal Processing etc. 